\title{
Carbohydrate counting
}

National Diabetes Information Clearinghouse (NDIC)

\section{Definitions}

Carbohydrate

Defined by National Diabetes Information Clearinghouse (NDIC)

Gram

Defined by National Diabetes Information Clearinghouse (NDIC)

Source

National Diabetes Information Clearinghouse (U.S.). (2009). The diabetes dictionary. [Bethesda, Md.]: U.S. Dept. of Health and Human Services, National Institutes of Health, National Institute of Diabetes and Digestive and Kidney Diseases, National Diabetes Information Clearinghouse.

A method of meal planning for people with diabetes based on counting the number of grams of carbohydrate in food. 